\title{
Numerical Solutions of Volterra Equations Using Galerkin Method with Certain Orthogonal Polynomials
}

\author{
James E. Mamadu' ${ }^{1}$ Ignatius N. Njoseh ${ }^{2}$ \\ ${ }^{1}$ Department of Mathematics, University of Ilorin, Ilorin, Nigeria \\ ${ }^{2}$ Department of Mathematics and Computer Science, Delta State University, Abraka, Nigeria \\ Email: mamaduebimene@hotmail.com, njoseh@delsu.edu.ng
}

Received 25 January 2016; accepted 23 February 2016; published 26 February 2016

Copyright (C) 2016 by authors and Scientific Research Publishing Inc.

This work is licensed under the Creative Commons Attribution International License (CC BY). http://creativecommons.org/licenses/by/4.0/

(c) (i) Open Access

\section{Abstract}

This work is aim at providing a numerical technique for the Volterra integral equations using Galerkin method. For this purpose, an effective matrix formulation is proposed to solve linear Volterra integral equations of the first and second kind respectively using orthogonal polynomials as trial functions which are constructed in the interval $[-1,1]$ with respect to the weight function $w(x)=1+x^{2}$. The efficiency of the proposed method is tested on several numerical examples and compared with the analytic solutions available in the literature.

\section{Keywords}

\section{Galerkin Method, Orthogonal Polynomials, Volterra Integral Equations}

\section{Introduction}

Modelling of physical problems arising from every discipline of study are often transformed into integral equations, namely, Volterra linear and nonlinear integral equations of the first and second kind respectively. For this, several authors have studied and applied these equations from the viewpoint of obtaining an analytic and unique numerical solution. In recent years, there has been a growing interest in the Volterra integral equations mainly due to its applicability in many areas of mathematical physics (astrophysics, contact problem, heat transfer problem and reactor theory). Consequently, most conventional analytic integral equations solvers have been developed and implemented since the digital computer was introduced some decades ago. The considerations are whether these solvers give an accurate solution, use less computation time, implement and give a compact solu- 
tion form.

However, most of these solvers such as the Adomial decomposition method (ADM), Laplace transform method (LTM) and the Successive substitution method (SSM) do not have solutions in compact form. Thus, numerical stimulation in engineering science and in applied mathematics has become a powerful tool to model difficult phenomena, particularly, when analytic solutions are difficult to achieve.

Many researchers have developed numerical methods for the solution of Volterra integral equations using various polynomials. Rahman [1] used Galerkin method with Hermite polynomial basis for the numerical solutions of Volterra integral equations of the second kind. Shafigul et al. [2] used Galerkin method to explore the solutions of linear and nonlinear Volterra equations using both Hermite and Chebychev polynomial basis. Shahsavaran [3] solved Volterra integral equations of Abel type using Block pulse functions. Maleknejad et al. [4] worked on a new approach to the numerical solution of Volterra Integrals by using Bernstain's approximation. Also, Kamyad et al. [5] worked on a numerical approach for solving equations with controlled error. Shirin and Islam [6] used these polynomials for solving Fredholm integral equations of the second kind. Amarantunga [7] described an augmented Galerkin technique for the solution of one dimension partial differential equation.

However, in this paper, an effective and efficient Galerkin numerical algorithm is formulated with orthogonal polynomials as basis which are constructed in the interval $[-1,1]$ with respect to the weight function $w(x)=1+$ $x^{2}$. The proposed method is employed to solve linear Volterra integral of the first and second kind with regular and weak singular kernels, in details, in Section 3. Section 2 presents the concept of orthogonal polynomials. Section 4 presents numerical experiments of different kinds of Volterra integral equations to verify the proposed method. The results of each numerical example indicate convergence and error analysis are discussed. Finally, the conclusion is presented in Section 5.

\section{The Orthogonal Polynomials}

Let

$$
\int_{a}^{b} w(x) \varphi_{i}(x) \varphi_{j}(x) \mathrm{d} x=h_{i} \delta_{i j}
$$

with the Kronecker delta $\delta_{i j}$ defined as

$$
\delta_{i j}= \begin{cases}0, & i \neq j \\ 1, & i=j\end{cases}
$$

where the weight function $\mathrm{w}(\mathrm{x})$ is continuous and positive on $[\mathrm{a}, \mathrm{b}]$ such that the moments

$$
\mu=\int_{a}^{b} w(x) x^{i} \mathrm{~d} x, i=0,1,2,3, \cdots
$$

exist.

Then the integral,

$$
\left\langle\varphi_{i}, \varphi_{j}\right\rangle=\int_{a}^{b} w(x) \varphi_{i}(x) \varphi_{j}(x) \mathrm{d} x
$$

denotes an inner product of polynomials $\varphi_{i}$ and $\varphi_{j}$.

For orthogonality,

$$
\left\langle\varphi_{i}, \varphi_{j}\right\rangle=\int_{a}^{b} w(x) \varphi_{i}(x) \varphi_{j}(x) \mathrm{d} x=0, i \neq j,[-1,1]
$$

If $\delta_{i j}=1$, then the polynomials are not only orthogonal but orthonormal.

In this study, we adopt the weight function $w(x)=1+x^{2}$ in the interval $[a, b] \equiv[-1,1]$

The construction of $\varphi_{i}, i=1,2,3, \cdots$ of the approximant:

$$
\tilde{y}(x)=\sum_{i}^{n} a_{i} \varphi_{i}(x) \cong y(x)
$$

now follows:

\section{Construction of Orthogonal Basis Function}

For the purpose of constructing the basis function, we use additional property that 


$$
\varphi_{n}(1)=1
$$

where

$$
\varphi_{n}(x)=\sum_{i=0}^{n} C_{i}^{(n)} x^{i}
$$

satisfies the orthogonality property (4).

Thus, the first six orthogonal polynomials $\varphi_{i}(x) ; i \geq 7$ valid in $[-1,1]$ are given below.

$$
\begin{gathered}
\varphi_{0}(x)=1 \\
\varphi_{1}(x)=x \\
\varphi_{2}(x)=\frac{1}{3}\left(5 x^{2}-2\right) \\
\varphi_{3}(x)=\frac{1}{5}\left(14 x^{3}-9 x\right) \\
\varphi_{4}(x)=\frac{1}{648}\left(333-2898 x^{2}+3213 x^{4}\right) \\
\varphi_{5}(x)=\frac{1}{136}\left(325 x-1410 x^{3}+1221 x^{5}\right) \\
\varphi_{6}(x)=\frac{1}{1064}\left(-460+8685 x^{2}-24750 x^{4}+17589 x^{6}\right)
\end{gathered}
$$

\section{Mathematical Formulation of Integral Equation}

In this section, we first consider the Volterra integral of the second kind given by

$$
y(x)+\lambda \int_{a}^{x} k(x, s) y(s) \mathrm{d} s=f(x), a \leq x \leq b
$$

where $y(x)$ is the unknown function to be determined, $k(x, s)$ is the kernel function, which is continuous or discontinuous integrable, $f(x)$ being the known function satisfying $f(a)=0$ and $\lambda$ is the constant.

Now, we use the Galerkin method to find an approximate solution $y_{n}(x)$ of Equation (7). Let the approximant be defined uniquely as Equation (5), where $\varphi_{i}(x)$ are orthogonal polynomials of degree $i$ constructed, $a_{i}$ are the unknown parameters to be determined and $n$ is the number of piecewise polynomials.

Now, substituting Equation (5) into Equation (7), we get

$$
\sum_{i=1}^{n}\left[\varphi_{i}(x)+\lambda \int_{a}^{x} k(x, s) \varphi_{i}(s) \mathrm{d} s\right]=f(x), a \leq x \leq b
$$

We obtain the Galerkin equation by multiplying both sides of Equation (8) by $\varphi_{i}(x), i=0(1) n$ and then integrating with respect to $x$ from $a$ to $x$ we obtain

$$
\sum_{i=1}^{n}\left[\int_{a}^{b}\left[\varphi_{i}(x)+\lambda \int_{a}^{x} k(x, s) \varphi_{i}(s) \mathrm{d} s\right] \varphi_{j}(x) \mathrm{d} x\right]=\int_{a}^{b} f(x) \varphi_{j}(x) \mathrm{d} x, j=0(1) n
$$

Equation (9) is written in the matrix form as

$$
A \underline{x}=\underline{b}
$$

where the elements of $A, \underline{x}$ and $\underline{b}$ are $a_{i j}, x_{i}$ and $b_{j}$ respectively, given by

$$
\begin{gathered}
a_{i j}=\left[\int_{a}^{b}\left[\varphi_{i}(x)+\lambda \int_{a}^{x} k(x, s) \varphi_{i}(s) \mathrm{d} s\right] \varphi_{j}(x) \mathrm{d} x\right], i, j=0(1) n \\
x_{i}=\left[x_{1}, x_{2}, \cdots, x_{n}\right]^{\mathrm{T}} \\
b_{j}=\int_{a}^{b} f(x) \varphi_{j}(x) \mathrm{d} x, j=0(1) n
\end{gathered}
$$

Now, the unknown parameters are determined with a solver, which in this case is the Gaussian elimination method, and substituting these parameters in Equation (5), we get the approximate solution $y(\tilde{x})$ of the 
integral Equation (7).

Now, we consider the Volterra equation of the first kind given by

$$
\int_{a}^{x} k(x, s) y(s) \mathrm{d} s=f(x), a \leq x \leq b
$$

where $y(x)$ is the unknown function to be determined, $k(x, s)$ is the kernel function, which is continuous or discontinuous integrable, $f(x)$ being the known function satisfying $f(a)=0$ and $\lambda$ is the constant.

Applying the same procedure as described above, we obtain the matrix form

$$
A \underline{x}=\underline{b}
$$

where the elements of $A, \underline{x}$ and $\underline{b}$ are $a_{i j}, x_{i}$ and $b_{j}$ respectively, given by

$$
\begin{gathered}
a_{i j}=\left[\int_{a}^{b}\left[\lambda \int_{a}^{x} k(x, s) \varphi_{i}(s) \mathrm{d} s\right] \varphi_{j}(x) \mathrm{d} x\right], i, j=0(1) n \\
x_{i}=\left[x_{1}, x_{2}, \cdots, x_{n}\right]^{\mathrm{T}} \\
b_{j}=\int_{a}^{b} f(x) \varphi_{j}(x) \mathrm{d} x, j=0(1) n
\end{gathered}
$$

The unknown parameters are determined with a solver, which in this case is the Gaussian elimination method, and substituting these parameters in Equation (5), we get the approximate solution $y(\tilde{x})$ of the integral Equation (14).

The absolute error for this formulation is defined by absolute error

$$
\left|y(x)-y_{n}(x)\right|
$$

\section{Numerical Examples}

To illustrate the effectiveness of the proposed method, we demonstrate the method with five numerical examples which include first and second kind with regular and weakly kernels. For all examples considered, the solutions obtained by the proposed method are compared with the exact solutions available in the literature. The rate of convergence of each of the Linear Volterra integral equations is composed as

$$
E_{r}=\left|\tilde{y}_{i+1}(x)-\tilde{y}_{i}(x)\right|<\delta
$$

where $\tilde{y}_{i}(x)$ is the approximate solution by the proposed method using the $n t h$ degree polynomial approximation and $\delta$ varies from $10^{-6}$ for $n \geq 10$ (See [2]).

Example 1: Consider the linear Volterra integral equation of the first with continuous kernel [2]

$$
\int_{0}^{x}(5+3 x-3 t) u(t) \mathrm{d} t=5 x^{2}+x^{3}, 0 \leq x \leq 1
$$

The exact solution is $u(x)=2 x$. Using the derived formula of Equation (15) and solving for $n \geq 1$, we get the approximate solution $\tilde{u}(x)=2 x$, which is the exact solution. This result agrees with [2] experiment with Chebychev polynomial basis for $n \geq 1$.

Example 2: Consider the first Abel's linear Volterra integral equation [2] of the form

$$
\int_{0}^{x} \frac{1}{\sqrt{(x-t)}} u(t) \mathrm{d} t=\frac{2}{\sqrt{105}} \sqrt{x}\left(105-56 x^{2}+48 x^{3}\right), 0 \leq x \leq 1
$$

The exact solution is $u(x)=x^{3}-x^{2}+1$. Results have been shown in table 1 for $n=2$. The maximum absolute errors obtained is in order of $10^{-4}$ for $n=2$. For $n=3$, we obtain the approximate solution as $\tilde{u}(x)=x^{3}-x^{2}+1$ which is the exact solution itself. On the other hand, the approximate solutions are same as exact solutions in the case of Chebychev polynomial basis for $n=10$ by [2] experiment. Also, the absolute errors were obtained in the order of $10^{-7}$ for $n=10$ by [4] with Bernstein's polynomials.

Example 3: Consider the second Abel's linear Volterra integral equation of the form [1]

$$
u(x)+\int_{0}^{x} \frac{1}{\sqrt{(x-t)}} u(t) \mathrm{d} t=x^{2}+\frac{16}{15} x^{\frac{5}{2}}, 0 \leq x \leq 1
$$


The exact solution is $u(x)=x^{2}$. Using orthogonal polynomials and derived formula in (10) for $n=2$, we get the approximate solution as $\tilde{u}(x)=x^{2}$, which is the exact solution. On the other hand, the absolute errors were obtained in the order $10^{-5}$ by [1] experiment with Hermite polynomials basis.

Example 4: Consider the first Abel's linear Volterra integral equation of the form [2]

$$
\int_{0}^{x} \frac{1}{\sqrt{(x-t)}} u(t) \mathrm{d} t=x^{r}, 0 \leq x \leq 1
$$

where $r$ is any positive number. The exact solution of the integral Equation (22) given by

$$
u(x)=\frac{2^{2 r-1}}{\pi} r \frac{(\Gamma(r))^{2}}{\Gamma(2 r)} x^{r-\frac{1}{2}}
$$

In one numerical example $r$ is chosen as $r=5$ and $\frac{3}{2}$.

For $r=5$, the exact solution is $u(x)=\frac{1280}{315} x^{\frac{9}{2}}$. Results have been shown in Table 2 for $n=2$ and 3 .

The maximum absolute errors obtain is in the order of $10^{-3}$ and $10^{-4}$ for $n=2$ and 3 respectively.

For $r=\frac{3}{2}$, the exact solution is $u(x)=\frac{3}{4} x$. Using the proposed method for $n \geq 1$, we obtain the approximate solution as $\tilde{u}(x)=\frac{3}{4} x$, which is the exact solution. This result is in line with [2] results with Hermite and Chebychev polynomial basis $n \geq 1$.

Example 5: Consider the second Abel's linear Volterra integral equation of the form [1]

$$
u(x)-\int_{0}^{x} u(t) \mathrm{d} t=\mathrm{e}^{x}, 0 \leq x \leq 1
$$

The exact solution is $u(x)=\mathrm{e}^{x}(1+x)$. Results is shown in Table 3 for $n=2$ and 3 . The maximum absolute errors obtain in the order for $10^{-3}$ and $10^{-4}$ for $n=2$ and 3 respectively. On the other hand, the absolute errors were obtained in the order $10^{-4}$ for $n=4$ by [1] experiment with Hermite polynomials basis.

\section{Conclusion}

In this paper, we have employed the Galerkin method based on the orthogonal polynomial basis tool which was constructed and has been developed to solve first and second kind Volterra integral equations. The numerical results obtained by the proposed method show an excellent rate of convergent even as $n$ increases, which is shown in Tables 1-5. Also, the numerical solutions coincide with the exact solutions even at few numbers of

Table 1. Computed Absolute Error of examples 1 for $n=2$.

\begin{tabular}{cccc}
\hline$x$ & Exact Solutions & Approximate Solutions & Absolute Error \\
\hline 0.00 & 1.0000000 & 1.0372960 & $3.7296 \mathrm{E}-02$ \\
0.10 & 0.9910000 & 0.9907925 & $2.0746 \mathrm{E}-04$ \\
0.20 & 0.9680000 & 0.9519814 & $1.6019 \mathrm{E}-02$ \\
0.30 & 0.9370000 & 0.9208625 & $1.6138 \mathrm{E}-02$ \\
0.40 & 0.9040000 & 0.9208625 & $6.5641 \mathrm{E}-03$ \\
0.50 & 0.8750000 & 0.8974359 & $6.7016 \mathrm{E}-03$ \\
0.60 & 0.8560000 & 0.8817016 & $1.7660 \mathrm{E}-02$ \\
0.70 & 0.8530000 & 0.8736597 & $2.0310 \mathrm{E}-02$ \\
0.80 & 0.8720000 & 0.8733100 & $8.6527 \mathrm{E}-03$ \\
0.90 & 0.9190000 & 0.8956876 & $2.3312 \mathrm{E}-02$ \\
1.00 & 1.0000000 & 0.9184149 & $8.1585 \mathrm{E}-02$ \\
\hline
\end{tabular}


Table 2. Computed Absolute Error of examples 4 for $n=2$.

\begin{tabular}{cccc}
\hline$x$ & Exact Solutions & Approximate Solutions & Absolute Error \\
\hline 0.00 & 0.0000000 & 0.0854492 & $8.5449 \mathrm{E}-02$ \\
0.10 & 0.0000409 & 0.0056061 & $5.5652 \mathrm{E}-03$ \\
0.20 & 0.0009255 & -0.0361694 & $3.7095 \mathrm{E}-02$ \\
0.30 & 0.0057385 & -0.0398773 & $4.5616 \mathrm{E}-02$ \\
0.40 & 0.0209421 & -0.0055176 & $2.6460 \mathrm{E}-02$ \\
0.50 & 0.0571629 & 0.0669098 & $9.7468 \mathrm{E}-03$ \\
0.60 & 0.1298465 & 0.1774048 & $4.7558 \mathrm{E}-02$ \\
0.70 & 0.2598309 & 0.3259674 & $6.6137 \mathrm{E}-02$ \\
0.80 & 0.4738648 & 0.5125977 & $3.8733 \mathrm{E}-02$ \\
0.90 & 0.8050833 & 0.7372955 & $6.7788 \mathrm{E}-02$ \\
1.00 & 1.2934497 & 1.0000610 & $2.9339 \mathrm{E}-01$ \\
\hline
\end{tabular}

Table 3. Computed Absolute Error of examples 4 for $n=3$.

\begin{tabular}{cccc}
\hline$x$ & Exact Solutions & Approximate Solutions & Absolute Error \\
\hline 0.00 & 0.0000000 & -0.0213623 & $2.1362 \mathrm{E}-02$ \\
0.10 & 0.0000409 & 0.0062002 & $6.1593 \mathrm{E}-03$ \\
0.20 & 0.0009255 & 0.0097061 & $8.7806 \mathrm{E}-03$ \\
0.30 & 0.0057385 & 0.0063387 & $6.0023 \mathrm{E}-04$ \\
0.40 & 0.0209421 & 0.0132812 & $7.6608 \mathrm{E}-03$ \\
0.50 & 0.0571629 & 0.0477171 & $9.4458 \mathrm{E}-03$ \\
0.60 & 0.1298465 & 0.1268295 & $3.0169 \mathrm{E}-03$ \\
0.70 & 0.2598309 & 0.2678019 & $7.9710 \mathrm{E}-03$ \\
0.80 & 0.4738648 & 0.4878174 & $1.3953 \mathrm{E}-02$ \\
0.90 & 0.8050833 & 0.8040594 & $1.0239 \mathrm{E}-03$ \\
1.00 & 1.2934497 & 1.2337112 & $5.9738 \mathrm{E}-02$ \\
\hline
\end{tabular}

Table 4. Computed absolute error of examples 5 for $n=2$.

\begin{tabular}{|c|c|c|c|}
\hline$x$ & Exact Solutions & Approximate Solutions & Absolute Error \\
\hline 0.00 & 1.0000000 & 1.0628855 & $6.2886 \mathrm{E}-02$ \\
\hline 0.10 & 1.2156880 & 1.2243500 & $8.6620 \mathrm{E}-03$ \\
\hline 0.20 & 1.4656833 & 1.4456876 & $1.9996 \mathrm{E}-02$ \\
\hline 0.30 & 1.7548164 & 1.7268981 & $2.7918 \mathrm{E}-02$ \\
\hline 0.40 & 2.0885546 & 2.0679816 & 2.0573E-02 \\
\hline 0.50 & 2.4730819 & 2.4689380 & $4.1439 \mathrm{E}-03$ \\
\hline 0.60 & 2.9153901 & 2.9297675 & $1.4377 \mathrm{E}-02$ \\
\hline 0.70 & 3.4233796 & 3.4504699 & $2.7090 \mathrm{E}-02$ \\
\hline 0.80 & 4.0059737 & 4.0310452 & $2.5072 \mathrm{E}-02$ \\
\hline 0.90 & 4.6732459 & 4.6714936 & $1.7523 \mathrm{E}-03$ \\
\hline 1.00 & 5.4365637 & 5.3718149 & $6.4749 \mathrm{E}-02$ \\
\hline
\end{tabular}


Table 5. Computed absolute error of examples 5 for $n=3$.

\begin{tabular}{cccc}
\hline$x$ & Exact Solutions & Approximate Solutions & Absolute Error \\
\hline 0.00 & 1.0000000 & 0.9945330 & $5.4670 \mathrm{E}-03$ \\
0.10 & 1.2156880 & 1.2167930 & $1.1050 \mathrm{E}-03$ \\
0.20 & 1.4656833 & 1.4679686 & $2.2853 \mathrm{E}-03$ \\
0.30 & 1.7548164 & 1.7556649 & $8.4845 \mathrm{E}-04$ \\
0.40 & 2.0885546 & 2.0874877 & $1.0669 \mathrm{E}-03$ \\
0.50 & 2.4730819 & 2.4710422 & $2.0398 \mathrm{E}-03$ \\
0.60 & 2.9153901 & 2.9139341 & $1.4560 \mathrm{E}-03$ \\
0.70 & 3.4233796 & 3.4237689 & $3.8930 \mathrm{E}-04$ \\
0.80 & 4.0059737 & 4.0081521 & $2.1784 \mathrm{E}-03$ \\
0.90 & 4.6732459 & 4.6746893 & $1.4434 \mathrm{E}-03$ \\
1.00 & 5.4365637 & 5.4309860 & $5.5777 \mathrm{E}-03$ \\
\hline
\end{tabular}

polynomials employed to find the approximate solution. Thus, the method is effective, efficient and reliable for the solution of other integral equations of other types.

\section{References}

[1] Rahman, M.M. (2013) Numerical Solutions of Volterra Integral Equations Using Galerkin Method with Hermite Polynomials. Proceedings of the International Conference on Applied Mathematics and Computational Methods in Engineering.

[2] Islam, S. and Rahman, A. (2013) Solutions of Linear and Nonlinear Volterra Integral Equations Using Hermite and Chebychev Polynomials. International Journal of Computers and Technology, 11.

[3] Shahsavaran, A. (2011) Numerical Approach to Solve Second Kind Volterra Integral Equations of Abel Type Using Block-Pulse Functions and Taylor Expansion by Collocation Method. Applied Mathematical Sciences, 5, 685-696.

[4] Maleknejad, Hashemizadeh, E. and Ezzati, R. (2011) A New Approach to the Numerical Solution of Volterra Integral Equations by Using Bernstein's Approximation. Communications in Nonlinear Science and Numerical Simulation, 16, 647-655. http://dx.doi.org/10.1016/j.cnsns.2010.05.006

[5] Kamyad, A.V., Mehrabinezhad, M. and Saberi-Nadjafi, J. (2010) A Numerical Approach for Solving Linear and Nonlinear Volterra Integral Equations with Controlled Error. IAENG, International Journal of Applied Mathematics, 40, 71-76.

[6] Shirin, A. and Islam, M.S. (2010) Numerical Solutions of Fredholm Integral Equations Using Bernstein’s Polynomials. Journal of Scientific Research, 2, 264-272.

[7] Amaratunga, K. (1994) Wavet-Galerkin Solution for One Dimension Partial Differential Equation. International Journal for Numerical Methods in Engineering, 37, 2703-2716. http://dx.doi.org/10.1002/nme.1620371602 